\title{
Contraceptive introduction and the management of choice: the role of Cyclofem in Indonesia
}

\author{
Ruth Simmons, ${ }^{*}$ Peter Fajans ${ }^{* *}$ and Firman Lubis $f$
}

${ }^{\star}$ Department of Population Planning and International Health and Department of Public Health Planning and Administration, School of Public Health, University of Michigan, Ann Arbor, MI 48109-2029, USA; * Department of Population Planning and International Health, School of Public Health, University of Michigan, Ann Arbor, MI; and TYayasan Kusuma Buana, Jakarta, Indonesia

This paper presents a programmatic perspective on the relationship between the introduction of new contraceptive technology and expanding contraceptive options, using the example of Cyclofem in Indonesia. Past approaches to contraceptive introduction have considered only the characteristics of the new method in the decision-making process. In assessing whether the introduction of a new method actually expands contraceptive choice for women and whether the program has the managerial capabilities to assure quality of care in this process, the authors argue that consideration must be given to all methods within a delivery system and how new technology relates to the management of contraceptive choice. Using this perspective, the authors suggest that choice would not necessarily be expanded with scaled-up service delivery of a new once-a month injectable in the Indonesian public sector context.

Keywords: monthly combined injectable contraception; Cyclofem; Indonesia; contraceptive choice

\section{Introduction}

A central rationale behind contraceptive development and introduction is choice: Presenting potential users with a range of options so that they

Address correspondence to: Dr. Ruth Simmons, Department of Population Planning and International Health and Department of Public Health Policy and Administration, School of Public Health, University of Michigan, Ann Arbor, MI 48109-2029, USA. 
can select a method best suited to their needs. However, whether or not users' options are actually broadened when new contraceptive technology is added to a service delivery system has not been widely examined. The assumption has been that addition of new technology by definition increases choice. Past approaches have defined the central issues of contraceptive introduction in terms of safety, user acceptability and continuation rates of a new method of contraception. Using the example of Cyclofem introduction in Indonesia, we argue that a shift is needed away from an exclusive focus on the new method to an evaluation of new technology as it interacts with the existing service delivery system and with user needs. Within this approach the guiding research questions are as follows: Does the introduction of a new method expand contraceptive options for the user and does the program have the managerial capabilities to assure quality of care in the process of adding new contraceptive technology to the existing method mix? This paper shows that an emphasis on programmatic factors rather than on the intrinsic characteristics of the method alone, dramatically alters conclusions about the impact of contraceptive introduction on choice.

With the growing emphasis on quality of care in family planning, ${ }^{1,2}$ the importance of assuring meaningful and safe contraceptive options has become a central theme in both the professional literature and the policy and program arenas. Meaningful choice implies variability in the intrinsic characteristics of methods, including such features as mode of administration, side-effects, duration and reversibility. New technology alone, however, does not broaden choice. The degree to which options are actually expanded depends on the existing method mix in a given setting, and on the program's capabilities to assure quality of care as new methods are added to the service delivery system. The most dramatic example of how the introduction of new technology by itself does not necessarily broaden choice is the history of intrauterine device (IUD) introduction in the 1960s, when the Lippes loop was hailed as the breakthrough in contraceptive technology that would solve India's population problem. Because of its easy reversibility, the IUD signified a major expansion of options, at a time when hormonal methods were not yet available in India, and the only medical method available was sterilization. Given the lack of attention to the social and cultural implications of the IUD for traditional women, and the failure to adapt the service delivery system to assure appropriate standards of technical care, medical backup and counseling, the IUD soon became discredited among Indian women as an acceptable contraceptive method.

In connection with the more recent introduction of Norplant and Cyclofem, so-called "introductory trials" have been organized upon completion of phase III clinical trials with the intention of assuring appropriate preparation of the service delivery system for the requirements of the 
new method. Closely modeled after the clinical trial in their research protocol, such introductory evaluations are intended to assess the safety, efficacy, acceptability and continuation rates of new technology under conditions that approximate routine service delivery, yet still benefit from special conditions. Given the additional inputs in supplies, staffing and training, and the relatively strong technical and managerial capabilities which are part of the selection criteria for introductory trial clinics, the addition of new technology is likely to broaden contraceptive options in these settings. Whether options are actually expanded, however, is usually not assessed. Introductory trial protocols are centered on outcome measures pertaining to a single method and not on the relationship of this method to existing contraceptive methods and the broader service delivery system. Moreover, since they do not represent typical service delivery settings, introductory trials shed little light on the impact of new contraceptive technology on choice within routine service delivery systems. Assessment of such impact requires research with a programmatic focus, placing new technology into the context of the existing method mix and the management capabilities of average service delivery settings. This paper presents findings from the Indoncsian Health Services Delivery Study on Cyclofem ${ }^{3}$, which adopted such a programmatic focus. We examine the relationship between the introduction of the once-a-month injectable and choice, showing that contraceptive options for Indonesian women would not necessarily expand with scaled-up service delivery of Cyclofem. A companion paper in this volume by Lubis and colleagues presents key findings on the technical quality of care and on the necessary adaptations in program management to ensure maintenance of appropriate technical standards of service. ${ }^{4}$

\section{The Indonesian Health Services Delivery Study: Background, Framework and Methods}

The Indonesian Health Services Delivery Study examined the implications of adding Cyclofem to the national family planning program while focusing on both quality of care and quality in management of the delivery system. At the time of the study's initiation, an introductory trial of Cyclofem was underway in six public sector health centers in Indonesia. The first objective of the study was to assess the managerial and quality of care implications of adding Cyclofem to these health centers. A sccond objective of the study was to assess the managerial requirements and/or adaptations that would be necessary for the introduction of Cyclofem on a nationwide basis. Studying non-trial settings provided an opportunity to predict the effect of Cyclofem introduction on both quality of care and on quality of management within these delivery contexts. Examination of whether other forms of contraception, particularly other injectables, 
were provided within a context of choice made it possible to anticipate the conditions of service delivery likely to characterize Cyclofem introduction within these same settings. The focus on quality of care and its managerial determinants is based on the argument that quality programs are likely to achieve high levels of adoption, use continuation and user satisfaction. ${ }^{5}$ For the purposes of this study, quality of care was defined as judgments about appropriate service delivery related to three critical areas: choice, interpersonal quality of care and technical quality of care. The present paper focuses on choice, raising the following questions: 1) Does Cyclofem introduction actually broaden contraceptive options for women, and 2) What are the managerial requirements of assuring such choice?

Assessing the managerial prerequisites of quality of care in the introduction of Cyclofem implies a need to develop contextual understandings and explanations of the service delivery system to which this new contraceptive will be added. Given the focus on management processes and implementation systems, a qualitative approach to data collection was chosen and combined with secondary quantitative data, to the extent that such data were available. Data collection consisted of systematic observation of service delivery, in-depth interviews and document reviews, and was conducted in the provinces where the introductory evaluations of Cyclofem were being conducted (Jakarta and West Java), as well as in West Sumatra, where the introductory trial was soon to be initiated as well. Observations were conducted in 28 service delivery sites, including: four of the six health centers involved in the introductory evaluation, seven non-trial health centers, four community-based health posts, and thirteen private sector settings. Non-trial health centers were selected purposely in each of the three provinces, in order to represent both urban and rural facilities, moderate resources and both average as well as weak program performance. Private sector settings consisted of physician or midwife practices or clinics of non-governmental organizations (NGOs). All methods provided in a given setting during the observation period were included in this study. A key characteristic of the observation technique in this study was to place the individual provider-client interactions into the context of the clinic session as a whole. The study instrument emphasized observation of patient flow through the clinic from the time a client approached the service delivery point to her exit from it. With this approach, 241 individual provider-client interactions were observed (Table 1). In addition to clinic observations, a total of 107 in-depth interviews (Table 2) were conducted with:

1. National leaders: in the National Family Planning Board (BKKBN, $)$ in the Ministry of Health (MOH), from the Indonesian Medical Association, the Indonesian Midwives' Association, the Association of Obste- 
TABLE 1. Number of provider-client interactions observed by type of family planning method and type of service delivery setting

\begin{tabular}{lcccccc}
\hline & Cyclofem & $\begin{array}{c}\text { DMPA, } \\
\text { NET-EN }\end{array}$ & IUD & Pill & Other* & TOTAL \\
\hline $\begin{array}{l}\text { Public sector } \\
\text { Jakarta }\end{array}$ & 7 & 18 & 9 & 17 & 2 & 53 \\
$\quad$ West Java & 19 & 51 & 9 & 3 & 2 & 84 \\
$\quad$ West Sumatra & na & 22 & 16 & 8 & 1 & 47 \\
Private sector & na & 13 & 8 & 7 & 4 & 32 \\
$\quad$ Jakarta & na & 7 & 11 & 3 & 2 & 23 \\
$\quad$ West Java & na & 0 & 1 & 1 & 0 & 2 \\
West Sumatra & 26 & 111 & 54 & 39 & 11 & 241 \\
TOTAL & & & & & & \\
\hline
\end{tabular}

*Other contraceptive methods used include: condoms, vaginal tablet, tubectomy, and vasectomy. na $=$ not applicable

tricians and Gynecologists, the Indonesian Planned Parenthood Association, the Blue Circle Program;

2. BKKBN and Ministry of Health leaders and program managers at the provincial and district level;

3. Public and private sector providers of services in introductory as well as non-trial sites, in urban, semi-urban and rural areas including BKKBN and $\mathrm{MOH}$ fieldstaff, and with providers at selected health centers, as well as with officials and reproductive health leaders at the provincial and national levels;

4. Community leaders as represented by village leaders, their wives and formal village women's groups;

5. Clients, including both Cyclofem acceptors and dropouts in trial areas, and users of other injectables and other methods in non-trial areas.

\section{Choice in Trial Centers and Future Potential for Choice in the Public Sector}

\section{Cyclofem Introduction in Trial Centers Increased Options for Some Women}

Information about the availability of contraceptive methods both at the community level and at health centers and health posts has been widely disseminated in Indonesia throughout the past several years. Family planning fieldworkers, their supervisors, village cadres, village leaders and a range of other community groups and institutions have been utilized to spread information about and mobilize demand for family planning 
TABLE 2. Number of interviews conducted

I. Number of Providers and Clients Interviewed by Province

\begin{tabular}{|c|c|c|c|c|c|c|c|c|}
\hline & \multicolumn{4}{|c|}{ PROVIDERS } & \multicolumn{3}{|c|}{ CLIENTS } & \multirow[b]{2}{*}{ TOTAL } \\
\hline & Doctor & Midwife & $\begin{array}{l}\text { Community } \\
\text { Leaders }\end{array}$ & $\begin{array}{c}\text { FP } \\
\text { Fieldstaff }\end{array}$ & $\begin{array}{l}\text { Cyclofem } \\
\text { Users }\end{array}$ & $\begin{array}{l}\text { Cyclofem } \\
\text { Drop-outs }\end{array}$ & $\begin{array}{c}\text { DMPA/ } \\
\text { NET-EN } \\
\text { Users }\end{array}$ & \\
\hline \multicolumn{9}{|l|}{ Jakarta } \\
\hline $\begin{array}{l}\text { Trial health centers } \\
\text { Non-trial health }\end{array}$ & 1 & 2 & 2 & 2 & 5 & 4 & 1 & 17 \\
\hline centers & 1 & 2 & - & - & na & na & - & 3 \\
\hline $\begin{array}{l}\text { Private sector } \\
\text { West Java }\end{array}$ & 2 & 1 & na & na & na & na & - & 3 \\
\hline $\begin{array}{l}\text { Trial health centers } \\
\text { Non-trial health }\end{array}$ & 5 & 3 & 1 & 1 & 5 & 4 & - & 19 \\
\hline centers & 1 & 1 & 3 & 3 & na & na & 4 & 12 \\
\hline Private sector & 3 & 2 & na & na & na & na & - & 5 \\
\hline West Sumatra* & & & & & & & & \\
\hline $\begin{array}{l}\text { Trial health centers } \\
\text { Non-trial health }\end{array}$ & 2 & 3 & - & - & na & na & - & 5 \\
\hline centers & 2 & 4 & 3 & 2 & na & na & 2 & 13 \\
\hline Private sector & 3 & 2 & na & na & na & na & - & 5 \\
\hline Total & 20 & 20 & 9 & 8 & 10 & 8 & 7 & 82 \\
\hline
\end{tabular}

II. Number of Program Officials and Professional Leaders Interviewed

\begin{tabular}{|c|c|c|c|c|c|}
\hline & BKKBN & $\begin{array}{l}\text { Ministry } \\
\text { of Health }\end{array}$ & $\begin{array}{l}\text { Professional } \\
\text { Leaders }\end{array}$ & TOTAL & \\
\hline National level & 9 & 3 & 4 & 16 & \\
\hline Provincial level & 5 & 4 & - & 9 & \\
\hline Total & 14 & 7 & 4 & 25 & \\
\hline \multicolumn{5}{|c|}{ GRAND TOTAL INTERVIEWED } & 107 \\
\hline
\end{tabular}

*These interviews were conducted in anticipation of inclusion of West Sumatra health centers in phase II of the Cyclofem introductory trial. na $=$ not applicable.

services. ${ }^{67}$ The Cyclofem introductory trial protocol, however, did not permit such promotional activities, thus information about this method within communities was limited.

In the Indonesian Family Planning Program, women identified as candidates for long term methods tend to be encouraged to use the IUD, occasionally sterilization, and more recently Norplant, while women wishing to space births are directed towards the pill and injectables. Cyclofem was typically introduced within the context of other injectable contraceptive service delivery. Women who came to the health center wanting an injectable or who were already using one were ottered the once-a-month 
injectable, particularly if they complained about amenorrhea or irregular bleeding, side-effects which are less common with Cyclofem. ${ }^{8}$ Introductory trial statistics confirmed that Cyclofem is predominantly provided to the subgroup of women who were either using an injectable or interested in using one. Forty-seven percent of Cyclofem users had switched from depot-medroxyprogesterone acetate (DMPA) use, 4 percent from norethisterone enantate (NET-EN) and Cyclofem, while 13 percent adoption was from pill users, 4 percent from the IUD, 4 percent from among other methods, and 29 percent from "new" contraceptive acceptors. ${ }^{y}$ In the introductoryide trial, new acceptors were defined as women not currently using a contraceptive. This includes both women who may have used one previously, including an injectable, and discontinued, and those who have never used a family planning method.

The introductory study of Cyclofem has expanded women's options in two respects. First, the intrinsic characteristics of the once-a-month injectable (regular bleeding patterns and quick return of fertility) provided additional variability to the existing contraceptive mix. Some women were given the opportunity to take advantage of this new option. Second, choice was expanded because women received a degree of information about the method not typically available in the Indonesian national program: written materials and information concerning benefits and pattern of follow-up. Possible side-effects, however, were not regularly mentioned. New acceptors of other methods receive only a limited amount of information from the health center staff. From a technical point of view, the quality of service delivery of Cyclofem also distinguished itself from other methods, as is discussed by Lubis et al. ${ }^{4}$

Nonetheless, expansion of choice in introductory trial settings was relatively modest. Within the public sector program, the availability of two other injectables placed clear limits on Cyclofem's contribution to the intrinsic variability of the contraceptive mix. The earlier addition of Norplant to the Indonesian program, by contrast, amounted to a significant expansion of method mix characteristics. In a Muslim society where sterilization or the pelvic examinations associated with IUD insertion have limited social and cultural acceptability, an additional long-acting method avoiding these social taboos has the potential of making a substantial contribution. Given the apparent rapid return to fertility, its easy reversibility, regular bleeding patterns, and high efficacy, Cyclofem has distinct advantages for young women, who would have reason for concern with the delay in return to fertility that usually characterizes DMPA. The advantages of the method for young women who wish to delay the onset of childbearing or to space the timing of childbirth were not given emphasis, however. Interest in the needs of the very young women at the beginning of their reproductive period is only now emerging as a policy emphasis of the Indonesian family planning program (Dr. Pandi, personal 
communication). At the time the fieldwork for this study was conducted, however, this interest had not yet been translated into patterns of service delivery at local health centers and trial health centers did not draw attention to Cyclofem's special relevance for young women.

The value of Cyclofem's more regular bleeding patterns was somewhat diminished by the availability of alternative options for addressing the frequent side-effects associated with DMPA use. Interviews with both providers and women established that DMPA users complaining about amenorrhea did not necessarily desire a regular monthly bleeding pattern, but were quite satisfied with an occasional period. Some providers broke the pattern of amenorrhea by stopping the injectable and providing one or two cycles of oral contraceptives, even though the possibility of inconsistent use of the pill exposed women to increased risk of pregnancy. A number of providers reported that with appropriate counseling at the time of the initial injection, side-effects of DMPA, especially amenorrhea, were frequently well tolerated. Such counseling, however, was not practiced regularly in the public sector program, as has also been noted in the literature. $6,7,10$

Of additional relevance to choice is the cost of contraception. In the introductory study, Cyclofem was provided without fee while other injectables were subject to varying degrees of locally levied charges, or had to be purchased through a private pharmacy, in an attempt to shift services towards self-sustainability. Future use of Cyclofem within the national program would involve similar charges. With the more frequent returns to the health center for services, user costs for Cyclofem in terms of transportation, time loss and other opportunity costs would be higher than those for the other injectables

\section{The Availability of NET-EN and DMPA in Routine Service Delivery has not Expanded Choice}

Two injectables are currently available in the Indonesian National Family Planning Program: DMPA and NET-EN. Both of these are progestogenonly contraceptives, which are often associated with disruption of normal menstrual bleeding, resulting in either amenorrhea or spotting, $, 8,1 ! \mathrm{DMPA}$ is administered every three months and NET-EN initially every two months and after the third injection, every three months. Injectable contraceptives were introduced into the government program in the late 1970 s, having been available several years earlier in the private sector and through special projects. At the time of this study, DMPA was the Inust widely used injectable. The general consensus among managers and providers was that injectables are a highly favored method of contraception because injections are generally well liked and associated with effective medical care. According to the 1991 Indonesian Demographic and 
Health survey, 11.7 percent of currently married women were using DMPA or NET-EN, making injectables the third most popular contraceptive method in Indonesia after the pill and the IUD (14.8 and 13.4 percent, respectively).

Even though NET-EN and DMPA are very similar to each other, the two injectables are distinct in their pattern of side-effects. ${ }^{8}$ Discontinuation rates due to irregular bleeding are the same for both injectables, while discontinuation rates for amenorrhea are lower for NET-EN. ${ }^{11}$ Examining how the availability of two injectables in the current program has affected women's choices, provides insight into the likely conditions of Cyclofem service delivery, if the method were introduced on a large scale. It was consistently observed that the differences between these two injectables were not emphasized to prospective clients. The practice was to substitute NET-EN for DMPA when stock depletion or logistics bottlenecks led to unavailability of DMPA. Typically, patients were not informed of the substitution. When women noticed the difference, providers reassuringly proclaimed there was none, except that an initial return visit was required in two instead of three months. Providers dislike NET-EN because it is an oil-based solution and, therefore, more difficult to inject. Since the program did not provide the appropriate bore needles for NET-EN, the injection was made unnecessarily cumbersome for the provider, given the difficulty of injecting the more viscous solution through a smaller bore needle. Under these conditions, injection of NET-EN is also more painful for the user. NET-EN and DMPA were supplied to public sector providers without regard to local demand or peripheral supplies such as needles. Although the preferred injectable was DMPA, intermittently health centers received, stocked and supplied only NET-EN. Thus, the existence of two progestogen-based injectables had not increased choice and, in fact, had reduced it in those cases in which women do not receive their preferred injectable because another has been substitutcd.

The pattern observed for NET-EN and DMPA was similar for IUDs as well. Of the three different IUDs available in the public sector program, at least two, the Multiload and the Lippes loop, were typically in stock at the health center. The Copper $T$ was less frequently available. In discussions with the client, and subsequent decision-making about IUD adoption, the differences among IUDs were rarely identified. Providers did not consider systematically which type of IUD would be most appropriate for each individual woman. For example, we observed a midwife who after insertion of the Multiload IUD realized that the woman had previously used an IUD for eight years and did not want any more children. The midwife commented to the observer: "Oh, I should have inserted a Lippes loop IUD, so she doesn't have to change it after four years."

Cyclofem, on the other hand, was generally distinguished from the other injectables both in the minds of the provider and the client. Its 
introduction had, therefore, expanded injectable choice in introductory study centers. Choice was also expanded in that Cyclofem's intrinsic characteristics are notably different from those of the progestogen-based injectables, although as mentioned above, the additional contribution to the expansion of the method mix was modest. Unless the service delivery system informs users of the differences among methods, and of each method's relative advantages and disadvantages, and assures consistent and affordable supplies of each injectable, choice will remain constrained. Findings on service provision of DMPA and NET-EN under conditions of routine service delivery do not bode well for the integration of an additional injectable, unless a major effort is to be made to differentiate the new technology from the other methods through counseling, informationgiving and better management of logistics and supplies.

\section{Policy and Managerial Constraints Impeding Expansion of Choice in the Public Sector}

Several managerial and policy barriers restrict choice in the Indonesian program and are likely to hamper effective service expansion of Cyclofem as well.

\section{Emphasis on Long-acting Methods}

During recent years, the Indonesian Family Planning Program has moved toward greater emphasis on long-term contraceptive methods, including the IUD, Norplant and sterilization. ${ }^{10,12}$ This policy translates into specific targets at all program levels, profoundly affecting service delivery. ${ }^{7}$ Acceptance of long-acting methods, and especially of the IUD, was highly valued by health center providers, subverting in several instances the objectives of the Cyclofem introductory study, and those relating to choice more generally. For example, we observed a woman who went to the midwife asking for family planning. The midwife asked: "Do you want the IUD or the implant?" The woman answered: "No, I don't, I want the injectable". Whereupon the midwife commented to the observer: "It is difficult. If the patient already wants the injectable, it is difficult to change her mind".

Emphasis on long-acting methods was observed consistently in the public sector both in trial and non-trial health centers. With this overriding concern, Cyclofem was not mentioned to women who came to the health center asking for the IUD, while requests for injectables were countered with the suggestion to use a long-term method. This tendency has also been noted in Pariani's study of contraceptive choice in East Java. ${ }^{13}$ Such emphasis on long-acting methods did not translate into refusal to accommodate women's initial requests, however. Women tended to leave the health center with the method to which they had declared an interest upon arrival. 
De-emphasis on Injectables in the Public Sector through Curtailed Supplies and Cost Recovery Efforts

Injectables within the public sector were furthermore de-emphasized through cost recovery and limited supplies. The intention was to shift the financial burden of injectables increasingly to the private sector or to seek cost recovery within the public sector health center. Clients were charged variable rates for injectables (with the exception of Cyclofem), while other contraceptives were provided free of charge. Also, mentioned by providers in several instances, was the National Family Planning Board's practice of supplying less than the needed number of injectables to health centers.

\section{Lack of Differentiation between Injectables in Record Keeping and Logistics Systems}

Certain features of the record keeping system impede available injectable options. Both DMPA and NET-EN are recorded under the category "suntikan", which translates into "injectable". Thus, the record keeping system cannot provide information on the number of users of each injectable, nor can the logistics system adjust the supply to clinic-based information on levels of demand. The administrative burden of adjusting a large, complex national program to the requirements of new contraceptive modalities, as well as inadequate awareness of the quality of care implications of such failure, are likely explanations for this lack of differentiation.

\section{Inadequate Emphasis on Information Giving and Counseling}

The concept and philosophy of counseling are now receiving somewhat greater attention in the Indonesian program than in the past. However, in terms of general program implementation, there is currently no culture which asks questions, or emphasizes the importance of listening to women's needs and expression of feelings, or encourages women to make their own decisions. ${ }^{6,7,10,14}$ The expectation that exchanges between providers and clients are to be brief is so well understood that women refrain from raising their concerns, knowing that providers answer questions in only the most perfunctory manner.

Information concerning the range of family planning options available and their respective advantages and disadvantages was not emphasized in service delivery. Program representatives often claimed that this function is performed through community-based program agents, while women typically reported that decisions were made based on the experience of friends and neighbors. Without attention to the information-giving component of care in clinic services, the meaningfulness of available contraceptive options is severely undermined. 
Burden on Staff Time

Availability and utilization of staff time constitute an additional impediment to the expansion of choice in the event of nationwide introduction of Cyclofem. Health center staff spent limited amounts of time with each client to assure speedy services to all patients, and in order to complete clinic sessions within the morning hours, typically considered available for clinic services. Introduction of Cyclofem would increase monthly patient loads in comparison with the amount of time required for reinjection of patients using a three-monthly injectable. Such increased pressure for service delivery does not enhance informed choice.

\section{Potential for Cyclofem in the Private Sector}

How appropriate are once-a-month injectables for the private sector? In Indonesia, the private sector is more varied than the public sector, consisting of practices of obstetricians and gynecologists, midwives, generalist private practitioners, and clinics organized by non-governmental organizations. Findings consistently indicate a considerable degree of choice and information-giving in the private sector. In contrast to the public sector, where women generally expressed a firm contraceptive preference, women using the private sector often had not made up their minds about which contraceptive to use. They tended more often to ask the provider for advice, or even to make the decision on their behalf. Private providers did not hesitate to offer their own opinion, but unlike the pattern in the public sector, such advocacy was based on patient needs, rather than on policy directives. Doctors and NGOs were observed to present fairly balanced and accurate technical information. Midwives in rural areas, however, conducted extensive conversations with women, with a focus on building trust and showing empathy, but transferal of correct factual information was limited.

NGO clinics offered a full range of contraceptive options, but individual private practices focused on a more limited set of methods, dictated by the training, technical capabilities and professional interest of the provider. Most frequently, private providers mentioned only those methods available in their practice or clinic, but left it up to the client to choose what she considered the most appropriate method. Providers differentiate among the types of IUDs, oral pills and injectables, although most chose not to provide NET-EN because of its perceived drawbacks (e.g. difficulty of procuring appropriate needles, difficulty and painfulness of administration).

Cyclofem would be introduced within a context of choice within the private sector, provided private practitioners like the method. It cannot be assumed that they will decide to stock the method, however. The cost of Cyclofem, its accessibility and the level of client demand are likely to 


\section{Role of Cyclofem in Indonesia: Simmons et al.}

determine whether or not they do so. Certainly, the frequency of client visits and fewer side-effects would be attractive features for private practitioners. The importance of Cyclofem in expanding choice is primarily its ability to avoid the bleeding irregularities that frequently accompany DMPA. In the private sector this contribution is, however, of somewhat lesser importance as providers indicated that good counseling typically overcomes women's discomfort with amenorrhea.

\section{Conclusions: Implications of Cyclofem Introduction for Choice in the Indonesian Program}

The relationship between choice and the introduction of new contraceptive technology is complex. Many factors determine what range of options exist for those who wish to limit or space childbearing. The technology itself, with its specific intrinsic characteristics, is only one among several determinants. Choice is a function of the new method, the other existing methods, the needs of users, and of the managerial capability of the service delivery system to assure both technical and interpersonal quality of care. Findings presented in this paper reveal that although Cyclofem introduction has broadened women's choice to some extent in the introductory trial settings, conditions of routine service delivery reveal a range of weaknesses likely to negate the potential contribution of an additional injectable.

Before discussing these implications further, the generalizability of study findings to the Indonesian Family Planning Program as a whole must be addressed. While the total number of study sites is relatively small, the sites include four of six Cyclofem trial centers, and taken as a whole, over-represent health centers and regions with strong management and service delivery capabilities. The fact that a variety of weaknesses in routine service delivery were observed in both the trial and non-trial health centers suggests that these weaknesses are present on a larger scale. Moreover, many of the identified constraints appear to be program constants rather than variables. For example, the push towards longacting methods, the de-emphasis of injectables generally within the public sector, the inability to distinguish between NET-EN and DMPA in the record keeping system, and the inattention to information-giving and counseling are all program constants well known to the family planning community in Indonesia. Furthermore, observations were corroborated by interviews with high level Ministry of Health and BKKBN officials, and documented in other reports. $6,7,10,12,13,15$

Thus, we feel confident in our conclusion that if the introduction of a once-a-month injectable is to expand women's options in Indonesia, considerable adaptations in operational policy and program management would be necessary. Changes would be required in counseling and infor- 
mation-giving, in the technical dimensions of care (as discussed by Lubis et al. ${ }^{4}$ in this volumel, the training of staff, supervision, record keeping systems, the provision of appropriate logistics and supplies, and in policy support for expanded options rather than a more narrow emphasis on long-acting methods. Given the scale and complexity of the Indonesian National Family Planning Program, such adaptations amount to both a significant organizational burden and to a change in direction which policy makers may be unwilling to pursue.

The introductory trial of Cyclofem in Indonesia occurs at a time when the family planning program is coping with the managerial implications of the introduction and scaled-up service delivery of Norplant. As Ward and colleagues indicated, ${ }^{15}$ Norplant introduction suffered from weaknesses in regard to informed choice, access to removal on demand and technical quality of care. The service delivery system was not capable of adjusting to the requirements of this new technology at the pace with which introduction proceeded, thereby jeopardizing not only the principle of informed choice, but other dimensions of the quality of care. Introduction of Cyclofem in Indonesia at this point in time would put added stress on an already overburdened health care system.

Maintaining or improving quality of care in the delivery of existing contraceptive methods is an additionally important consideration for the introduction of new methods. The introductory study of Cyclofem in Indonesia coincides with an increasing recognition among senior policy makers and program managers of the weaknesses in the quality of service delivery of existing program methods, especially in regard to long-acting methods. In some program settings, such improvements in quality of care might provide economies of scale for the introduction of new methods, as the necessary administrative and service delivery adjustments or even the training could incorporate the requirements for the new method as well. Given the limits in organizational capacity and resources, however, incremental approaches focused on improvements in the existing method mix may be preferred, particularly if the properties of the new technology do not greatly expand the range of options available to women.

The costs of adapting service delivery systems to the requirements of new technology may be extensive, especially in countries with the scale and complexity of the Indonesian program. The introductory study of Cyclofem in Indonesia takes place at a time when the program must move towards financial self-sufficiency. Civen this and other priorities, the resources for the necessary managerial and health service adaptation may not be available to assure quality of care and choice in the introduction of Cyclofem. Selective introduction of Cyclofem into urban areas, or certain regions, might be appropriate from the perspective of both costs and demand for the method. Selective introduction of new methods within public sector programs, however, is often not a politically acceptable 
alternative. Policy makers are hesitant to pursue this route, implying that Cyclofem should either be introduced on a national scale or not at all.

Selective introduction into the private sector, or within the social marketing program, on the other hand, is another alternative to be considered. Findings from this study indicate that categorical assumptions about high quality of care in the private sector are unwarranted. The private sector in Indonesia exhibits diverse quality of care characteristics. Some of the institutions are equipped to handle the service delivery adaptations to once-a-month injectables without major difficulty, provided they receive support with training and instructional materials. Other segments of the private sector-especially private providers in rural areas-are much less likely to provide such new technology with appropriate levels of quality of care, without significant program inputs from the public sector.

Health service delivery systems of other family planning programs in many other countries of the non-industrialized world are similar to the service delivery system in Indonesia. In fact, the Indonesian program compares favorably in terms of its administrative capabilities to many other developing country programs. In such settings, the addition of new technology must be considered with much caution. When programs do not manage to provide existing contraceptives with appropriate standards of care, the addition of new technology does not expand choice. These points are particularly important for those settings in which injectables already exist. In fact, health service delivery research in Bangladesh has concluded that quality of care would be improved if the Bangladesh national program would withdraw NET-EN from the program, leaving only one injectable within the system. ${ }^{16}$ This recommendation argues that such removal would, in fact, improve quality of service delivery.

If new contraceptive technology is to expand choice, careful assessments of the existing method mix, of the service delivery context must be undertaken. In countries with relatively stronger service delivery systems, or with the resources to adapt to the requirements of once-a-month injectables, the conditions for effective broadening of choice might exist. The potential for increased choice would be particularly great in settings where injectables are not currently available, or where, as in several countries in Latin America, Cyclofem could replace a widely used high-dose once-a-month injectable. The improved characteristics of a low-dose oncea-month injectable certainly increases women's options. $\Lambda$ dditionally, a once-a-month injectable such as Cyclofem holds promise for very young women desiring rapid return to ovulation. In fact, Indonesia might well be a case in which Cyclofem could find a niche among this population. However, without the requisite conditions in service delivery management, the addition of a once-a-month injectable does not enhance choice, but threatens to diminish it. 


\section{Acknowledgments}

This study was implemented by Yayasan Kusuma Buana (YKB), an Indonesian research and service organization, on behalf of the Indonesian $\mathrm{Na}$ tional Family Planning Board (BKKBN), with financial support from the Special Programme of Research, Development and Research Training in Human Reproduction. The authors would like to thank Dr Astrid Sulistomo and Ms Subadra Indrawati for their invaluable assistance in the collection and analysis of the data, as well as Dr Srihartati Pandi of BKKBN and Dr Nardho Gunawan of the Ministry of Health, and other members of the steering committee for their assistance and support. Ms Leslie Gray provided additional assistance with preparation and editing of the manuscript.

\section{References}

1. Bruce J. Fundamental elements of quality of care: A simple framework. Stud Fam Plann 1990;13:44-58.

2. Jain A, Bruce I, Kumar S. Quality of Services, Programme efforts and fertility reduction. In: Phillips I and Ross J, eds. Family Planning Programmes and Fertility. Oxford: Clarendon Press, 1992:202-21.

3. Lubis F, Fajans P, Sulistomo A, Indrawati S, Simmons R. Service delivery implications of introducing Cyclofem in Indonesia. 1992 (report submitted to BKKBN).

4. Lubis F, Fajans P, Simmons R. Maintaining technical quality of care in the introduction of Cyclofem in a national family planning program: Findings from Indonesia. Contraception 1994;49:527-541.

5. Jain A. Fertility reduction and the quality of family planning services. Stud Fam Plann 1989;16:181-98.

6. World Bank. The World Bank and Indonesia's Population Program. Report No. 9370. Operations Evaluation Department, 1991.

7. Warwick D. The Indonesian Family Planning Program: Government influence and client choice. Popul Develop Rev 1986;12:453-90.

8. Liskin LS. Long-acting progestins. Promise and prospects. Popul Reports 1983;11:K17-K55.

9. Hall $P$ and the Task Force on Research on Introduction and Transfer of Technologies for Fertility Regulation. The introduction of Cyclofem into national family planning programs: Experience from studies in Indonesia, Jamaica, Mexico, Thailand and Tunisia. Contraception 1994;49:489-507.

10. Smyth I. The Indonesian Family Planning Program: A success story for women? Development and Change 1991;22:781-805.

11. World Health Organization. Injectable contraceptives: their role in family planning care. Geneva, 1990.

12. Lerman C, Molyneaux J, Moeljodihardjo S, Pandjaitan S. The correlation between family planning program inputs and contraceptive use in Indonesia. Stud Fam Plann 1989;20:26-37.

13. Pariani S, Heer D, Van Arsdol M Jr. Does contraceptive choice make a differ- 
Role of Cyclofem in Indonesia: Simmons et al.

ence to contraceptive use? Evidence from East Java. Stud Fam Plann 1991;22:384-90.

14. Widyantoro N. Understanding patients' needs. People 1989;16:23.

15. Ward S, Poernomo I, Simmons R, Simmons G. Service delivery systems and quality of care in the implementation of NORPLANT in Indonesia, 1990 (report submitted to The Population Council).

16. International Centre for Diarrhoeal Disease Research, $\mathrm{MCH}-\mathrm{FP}$ Extension Project Briefing Paper No. 16, March 1991. 